\title{
PAPER
}

Check for updates

Cite this: Soft Matter, 2018, 14,8137

\section{Synergistic actions of mixed small and large pores for capillary absorption through biporous polymeric materials}

\author{
Thibault Lerouge, ${ }^{\mathrm{ab}}$ Olivier Pitois, (D) *a Daniel Grande, (D) ${ }^{\mathrm{b}}$ \\ Benjamin Le Droumaguet (D) $^{b}$ and Philippe Coussot (D) ${ }^{a}$
}

\begin{abstract}
Water absorption in porous media is an important process involved in numerous materials for various applications, such as in the building industry, food processing and bioengineering. Designing new materials with appropriate absorption properties requires an understanding of how absorption behavior depends on both the material's morphology and the properties of the solid matrix, i.e. hydrophilic/ hydrophobic nature and swelling/deformation properties. Although the basic principles of imbibition are well-known for simple porous systems, much less is known about absorption in complex porous systems, in particular those containing several coexisting porous phases, such as wood for example. Here, water absorption is studied for model porous organic materials exhibiting several degrees of hydrophobicity and two pore size levels, either as monoporous materials (large or small pores) or as biporous materials (mixed large and small pores). The interconnected biporous structure is designed via a double porogen templating approach using cubic sodium chloride particles as templates for the generation of the larger pore size $(250-400 \mu \mathrm{m})$ and $\mathrm{i}-\mathrm{PrOH}$ as a porogenic solvent for the smaller pore size $(2-5 \mu \mathrm{m})$. While absorption for the small pore material is well described by the classical Washburn theory, the large pore material shows a drastic reduction in the imbibition rate. This behavior is attributed to the slow breakthrough mechanism for the water interface at sharp edge connections between pores. Remarkably, this slow regime is suppressed for the biporous material and the imbibition rate is even higher than the sum of rates obtained for its monoporous counterparts, which highlights the synergistic action of mixed small and large pores.
\end{abstract}

Received 9th July 2018,

Accepted 1st October 2018

DOI: $10.1039 / \mathrm{c} 8 \mathrm{sm} 01400 \mathrm{k}$

rsc.li/soft-matter-journal

\section{Introduction}

Water imbibition in porous materials is a crucial phenomenon for numerous applications. Consider the building industry for example, where durability of the materials can be jeopardized by successive imbibition/drying cycles. In such a case water repellents can be used for preventing or decreasing water absorption. ${ }^{1}$ Water absorption is important for food processing (rehydration) and preservation, ${ }^{2}$ as well as for the basic process underlying ink writing, where liquids spread on a rough porous substrate, ${ }^{3}$ and for microfluidics. ${ }^{4}$ Theory of capillary imbibition has been intensively studied since the pioneering works of Bell \& Cameron, ${ }^{5}$ Lucas $^{6}$ and Washburn. ${ }^{7}$ They assumed, in agreement with experimental observations, that under the

\footnotetext{
${ }^{a}$ Université Paris Est, Laboratoire Navier, UMR 8205 CNRS - Ecole des Ponts ParisTech-IFSTTAR, 5 bd Descartes, 77454 Marne-la-Vallée Cedex 2, France. E-mail: olivier.pitois@ifsttar.fr

${ }^{b}$ Université Paris-Est, Institut de Chimie et des Matériaux Paris-Est (ICMPE), UMR 7182 CNRS-UPEC, 2 rue Henri Dunant, 94320 Thiais, France
}

action of capillary effects (Laplace pressure below the interface) a wet front progresses through the sample while saturating the material behind. In this context a straight front allows minimizing surface energy. The basic theory has been modified for including inertial and gravitational forces, ${ }^{8}$ pore shape and constriction effects. ${ }^{9-11}$ Besides, various other works focused on the possible roughness of the front ${ }^{12}$ or front broadening, ${ }^{13}$ associated with specificities of the porous structure.

The above basic theory turns out to be clearly inappropriate when applied to complex materials with several levels of porous phases. This is the case for foamed concretes ${ }^{14}$ or woods ${ }^{15}$ for example, in which effects of multimodal distributions of interconnected pores remain difficult to understand in terms of material's sorption characteristics. However, designing new materials with appropriate sorption properties requires an understanding of how sorption behavior depends on both the material's morphology and the properties of the solid matrix, i.e. hydrophilic/hydrophobic nature, swelling properties, etc.

In order to progress in the understanding of absorption characteristics of such complex media, we devised model porous 
organic materials exhibiting several degrees of hydrophobicity and two pore size levels, either as monoporous materials (large or small pores) or as "biporous" materials (i.e. with a mixture of the two porous phases). The interconnected biporous structure is formed via a double porogen templating approach using cubic sodium chloride particles as macroporogens for the creation of the larger pore size $(250-400 \mu \mathrm{m})$ and $\mathrm{i}-\mathrm{PrOH}$ as a porogenic solvent for the smaller pore size $(2-5 \mu \mathrm{m})$. As shown in the present paper, even such a simple mixture of small and large pores induces interesting synergistic behaviors that cannot be easily anticipated from sorption properties of its monoporous counterparts. We start by presenting the materials and methods, then we look in detail at the characteristics of the devised media, and finally we study the absorption properties of the different media.

\section{Materials and methods}

\subsection{Materials}

2-Hydroxyethyl methacrylate (HEMA, 97\%), ethyleneglycol dimethacrylate (EGDMA, 98\%), and 2,2-dimethoxy-2-phenylacetophenone (DMPA, 99\%) were purchased from Sigma Aldrich. Eugenol (99\%) was obtained from Alfa Aesar. Sodium chloride ( $\mathrm{NaCl}$ ) particles with sizes ranging from 50 to $500 \mu \mathrm{m}$ were purchased from Prolabo, and were stored under moisture-free conditions. Prior to use, they were sieved to isolate the particle fraction with average sizes between 250 and $400 \mu \mathrm{m}$. Propan-2-ol (i-PrOH, for analysis, ACS-Reag.Ph.Eur), and dichloromethane (DCM) were supplied by Carlo Erba. All reagents and solvents were used without further purification. 18.2 $\mathrm{M} \Omega$ deionized water was filtered through a Milli-Q Plus purification pack.

\subsection{Synthesis and preparation of porous materials}

2.2.1. Synthesis of eugenyl methacrylate (EgMA) monomer. The synthesis of eugenyl methacrylate (EgMA) was achieved similarly to procedures reported earlier. ${ }^{16-18}$ In a three-necked round bottom flask equipped with a dropping funnel, eugenol (56.75 g, $244 \mathrm{mmol}, 0.9$ equiv.), triethylamine (59 mL, $425 \mathrm{mmol}$, 1.1 equiv.) and DCM $(100 \mathrm{~mL})$ were placed. The reaction mixture was cooled down to $0{ }^{\circ} \mathrm{C}$ with an ice/water bath. Methacryloyl chloride (40.41 g, $0.39 \mathrm{mmol}, 1$ equiv.) in solution in $50 \mathrm{~mL}$ of DCM was added dropwise at $0{ }^{\circ} \mathrm{C}$ under an inert atmosphere. After completion of the addition, the reaction mixture was allowed to warm to room temperature overnight. The medium was filtered off to remove triethylammonium chlorhydrate. The organic solution was washed with $100 \mathrm{~mL} 5 \% \mathrm{NaOH}$ aqueous solution, $3 \times 100 \mathrm{~mL}$ deionized water, and dried over $\mathrm{MgSO}_{4}$. Filtration afforded the crude product that was finally purified by distillation under reduced pressure.

${ }^{1} \mathrm{H}$ NMR $\left(\mathrm{CDCl}_{3}, 400 \mathrm{MHz}\right): \delta(\mathrm{ppm}) 6.97(1 \mathrm{H}, \mathrm{d}, J=7.9 \mathrm{~Hz}$, $\left.\mathrm{H}_{\mathrm{ar}}\right), 6.82-6.74\left(2 \mathrm{H}, \mathrm{m}, \mathrm{H}_{\mathrm{ar}}\right), 6.35(1 \mathrm{H}, \mathrm{s}, \mathrm{H}), 5.97(1 \mathrm{H}, \mathrm{ddt}$, $J=16.8,10.1 \& 6.7 \mathrm{~Hz}, \mathrm{H}), 5.77-5671(1 \mathrm{H}, \mathrm{m}, \mathrm{H}), 5.15-5.04$ $(2 \mathrm{H}, \mathrm{m}, \mathrm{H}), 3.81\left(3 \mathrm{H}, \mathrm{s}, \mathrm{CH}_{3}-\mathrm{O}-\right), 3.39(2 \mathrm{H}, \mathrm{d}, J=6.7 \mathrm{~Hz}$, $\left.\mathrm{CH}_{2}-\mathrm{Ph}\right), 2.07\left(\mathrm{CH}_{3} \mathrm{R}\right)$.
${ }^{13} \mathrm{C}$ NMR spectrum $\left(\mathrm{CDCl}_{3}\right): \delta(\mathrm{ppm}) 165.69(C=\mathrm{O}), 151.12$ $\left(\mathrm{C}_{\mathrm{ar}}^{\mathrm{IV}}\right), 138.97\left(\mathrm{C}_{\mathrm{ar}}^{\mathrm{IV}}\right), 138.27\left(\mathrm{C}_{\mathrm{ar}}^{\mathrm{IV}}\right), 137.23\left(\mathrm{CH}=\mathrm{CH}_{2}\right), 135.79$ $\left(\mathrm{CH}_{2}=\mathrm{C}-\mathrm{CH}_{3}\right), 127.15\left(\mathrm{CH}_{2}=\mathrm{C}-\mathrm{CH}_{3}\right), 122.66\left(\mathrm{CH}_{\mathrm{ar}}\right), 120.79$ $\left(\mathrm{CH}_{\mathrm{ar}}\right), 116.21\left(\mathrm{CH}=\mathrm{CH}_{2}\right), 112.91\left(\mathrm{CH}_{\mathrm{ar}}\right), 55.99\left(\mathrm{CH}_{3}-\mathrm{O}-\mathrm{Ph}\right)$, $40.21\left(\mathrm{CH}_{2}-\mathrm{Ph}\right), 18.60\left(\mathrm{CH}_{3}-\mathrm{C}=\mathrm{CH}_{2}\right)$.

ATR-FTIR $\left(\mathrm{cm}^{-1}\right): 1726\left(\nu_{\mathrm{C}=\mathrm{O}}\right), 1638\left(\nu_{\mathrm{C}=\mathrm{C}}\right), 1600-1508-$ $1462\left(\nu_{\mathrm{C}=\mathrm{C} \text { aromatic }}\right), 1415-1380\left(\delta_{\mathrm{C}=\mathrm{C}}\right), 856 \& 806\left(\delta_{\mathrm{CH}}\right.$ aromatic$)$, $1200 \& 1130$ ( $\left.\nu_{\mathrm{C}-\text { O methyl aryl ether }}\right), 1265 \& 1035$ ( $\left.\nu_{\mathrm{C}-\mathrm{O} \text { aryl ester }}\right)$.

2.2.2. Preparation of poly(2-hydroxyethyl methacrylate-coethyleneglycol dimethacrylate) biporous material. Roughly cubic NaCl particles were sieved with sieves of size between 400 and $250 \mu \mathrm{m}$. Then they were submitted to a Spark Plasma Sintering (SPS) treatment so as to obtain a fused $\mathrm{NaCl}$ particle phase. A Sumitomo Dr Sinter Lab 515S SPS machine from Fuji Electronic Industrial was used. $\mathrm{NaCl}$ particles were introduced into a $51 \mathrm{~mm}$ wide circular graphite die, under a $50 \mathrm{kN}$ force onto the sample, then heated from room temperature to $100{ }^{\circ} \mathrm{C}$ at a heating rate of $50{ }^{\circ} \mathrm{C} \min ^{-1}$ under an inert atmosphere. After $20 \mathrm{~min}$ at $100{ }^{\circ} \mathrm{C}$ and $50 \mathrm{kN}$, the sintered sample was cooled down to room temperature at a rate of $50{ }^{\circ} \mathrm{C} \mathrm{min}^{-1}$ and the force was released. The polymerization mixture, composed of HEMA, EGDMA (with a molar ratio of $70 / 30 \mathrm{~mol} \%$ ) and DMPA ( 2 wt\% with respect to the total co-monomer amount) was homogenized with $80 \mathrm{vol} \% \mathrm{i}-\mathrm{PrOH}$ (with respect to the total volume of co-monomers). This mixture was added within the sintered $\mathrm{NaCl}$ particle template under reduced pressure, and then placed in a spectrolinker XL-1500 UV oven (Spectronics, Westburry, NY, USA) equipped with six lamps $(6 \times 15 \mathrm{~W})$ for $4 \mathrm{~h}$ under irradiation at $365 \mathrm{~nm}$ so as to trigger the photo-induced free-radical copolymerization. Both porogenic agents were removed by immersing the resulting materials in deionized water for 2 days, and the extraction solution was renewed twice a day. The materials were finally abundantly washed with deionized water and dried under vacuum at room temperature overnight. The removal of porogenic agents was verified by gravimetric analysis (final weight of the material stable and equivalent to the total mass of comonomers in the initial polymerization mixture).

2.2.3. Preparation of poly(2-hydroxyethyl methacrylate-coeugenyl methacrylate-co-ethyleneglycol dimethacrylate) biporous material. To vary the hydrophobic/hydrophilic balance of HEMA-based porous materials, our attention was focused on a hydrophobic bio-based phenol derivative originating from cloves, i.e. eugenol methacrylate. A series of biporous materials (BP) mainly differing from the EgMA molar content - from 0 to $70 \mathrm{~mol} \%$ with respect to the total co-monomer content - was thus prepared in the presence of both porogenic agents (i.e. i-PrOH or sintered NaCl 3-D template). The hydrophilic HEMA monomer is gradually substituted by the eugenyl methacrylate (EgMA) monomer to maintain $30 \mathrm{~mol} \%$ of EGDMA and $70 \mathrm{~mol} \%$ of HEMA/EgMA. The copolymer formula can be written as $\mathrm{P}\left(\mathrm{EGDMA}_{30}-\mathrm{co}-\mathrm{HEMA}_{x}-\mathrm{co}-\mathrm{EgMA}_{y}\right)$, so we refer in the following sections to the percentage, \%HEMA $=x$, that represents HEMA $\mathrm{mol} \%$ with respect to the total co-monomer molar content.

2.2.4. Preparation of monoporous analogues. The monoporous analogue materials with only large pores (LP) or small 
pores (SP) were synthetized by using a single porogen agent: the $\mathrm{NaCl}$ template (LP) or the i-PrOH solvent (SP).

\subsection{Structural and morphological characterization}

2.3.1. Nuclear magnetic resonance (NMR). ${ }^{1} \mathrm{H}$ NMR analyses were carried out on a Bruker Avance II spectrometer operating at a resonance frequency of $400 \mathrm{MHz}$ at room temperature using $\mathrm{CDCl}_{3}$ as the deuterated solvent.

2.3.2. Infrared spectroscopy (IR). Infrared spectra were recorded using a Bruker Tensor 27 DTGS spectrometer in attenuated total reflection (ATR) mode between 4000 and $450 \mathrm{~cm}^{-1}$ with an average of 32 consecutive scans and a resolution of $4 \mathrm{~cm}^{-1}$.

2.3.3. Scanning electron microscopy (SEM). Scanning Electron Microscopy (SEM) analyses of the materials were performed with a Merlin microscope from Zeiss equipped with InLens and SE2 (secondary electrons) detectors using a low accelerating tension $(2-3 \mathrm{kV})$ with a diaphragm aperture of $30 \mu \mathrm{m}$. Prior to analyses, the samples were coated with a $4 \mathrm{~nm}$ thin layer of palladium/platinum alloy in a Cressington $208 \mathrm{HR}$ sputter-coater. Pore sizes were determined using the ImageJ $1.47 \mathrm{v}$ software.

2.3.4. Mercury intrusion porosimetry (MIP). The porosity ratios, pore volumes, and pore size distributions of the materials were determined by mercury intrusion porosimetry (MIP) using an AutoPore IV 9500 porosimeter from Micromeritics. The determination of the porosity features was based on the Washburn equation between the applied pressure (from 1.03 to $206.8 \mathrm{MPa}$ ) and the pore diameter into which mercury intruded.

2.3.5. Contact angle measurements. Contact angles were measured using a tensiometer (Teclis). Water drops were forced to spread from a syringe onto either solid polymer films or porous samples' surfaces. A CCD camera was used to obtain images (see Fig. 4) of the liquid/air interface during spreading. The measured contact angle is the advancing contact angle $\theta$ (solid polymer films) or $\theta_{\mathrm{p}}$ (porous material).

\subsection{Transport properties}

2.4.1. Permeability measurements. Permeability measurements were performed with a homemade system. Briefly, cylindrical porous polymeric samples (varying height $L$ and $16 \mathrm{~mm}$ diameter) were sealed with a thin film of Teflon inside a long cylindrical plexiglas tube. The tube was partially immersed in a water bath and the water level was maintained at a fixed position by allowing the water to overflow from the bath, so it was used as a reference for height measurements, i.e. $z=0$. The bottom of the sample was maintained at height $z_{1}<-L$, so the porous sample was saturated with water. Then an amount of water was introduced into the tube, corresponding to a water height $z$. Such a configuration drives the water to flow through the porous sample with volume flow rate $Q$, or equivalently, with a superficial velocity $V_{\mathrm{D}} \equiv \mathrm{d} z / \mathrm{d} t=Q / S$, where $S$ is the sample's cross-section. The driving pressure difference is $P=\rho g(z(t)-L)$ and the permeability is given by the Darcy law:

$$
k=\frac{\mu L}{\rho g(z(t)-L)} \frac{\mathrm{d} z}{\mathrm{~d} t}
$$

where $\rho$ and $\mu$ are respectively the density and the dynamic viscosity of water. Using eqn (1) the permeability was deduced from measurements of $z(t)$. Note that we checked that the water flow was characterized by low values of the Reynolds number.

2.4.2. Imbibition experiment. Imbibition through our porous polymeric materials was measured with a homemade system. The sample was hung under an analytical balance having the appropriate mass precision $(0.1 \mathrm{mg})$. A water bath was allowed to be put in contact with sample's bottom and the mass was recorded as a function of time, i.e. a curve $M(t)$. In the following, it is more convenient to consider the corresponding water height in the sample:

$$
h(t)=\frac{M(t)}{\rho S \phi}
$$

We recall that $\rho, S$ and $\phi$ are respectively the density of water, the cross-section area and the pore volume fraction of the samples. Note that a water meniscus forms from the bath at the bottom of the samples, so we have subtracted the corresponding capillary force (as measured at the end of the experiment when the imbibed sample was detached from the bath) from $M(t)$. This procedure could induce some artefact at an early stage of the imbibition process, when the meniscus is not yet completely formed. In addition, a camera was used to follow the progression of the water front at the sample's surface. In practice, light transmission through the sample allowed us to observe the imbibition process: imbibed parts appeared lighter (see Fig. 6).

\section{Results}

\subsection{Morphological characteristics of the porous materials}

The two distinct monoporous materials presenting the small pores (SP) or the large pores (LP) were obtained by DMPA photo-triggered radical copolymerization of HEMA functional monomers and EGDMA as the crosslinking agent in the presence of a porogenic agent. Scanning electron microscopy allowed for ascertaining the presence of interconnected pores of a few hundreds of $\mu \mathrm{m}$ in size when obtained from sintered 250-400 $\mu \mathrm{m} \mathrm{NaCl} \mathrm{particles} \mathrm{(Fig.} \mathrm{1a)} \mathrm{in} \mathrm{LP} \mathrm{samples.} \mathrm{Similarly,} \mathrm{an}$ interconnected porosity of approximately $1 \mu \mathrm{m}$ was observed by SEM for small-pore samples after the removal of the porogenic solvent (Fig. 1b). The observation of biporous materials (BP) displaying both large and small pores revealed the presence of polymer thin walls between both porosity levels, as shown in Fig. 2. Additionally, numerous narrow interconnections, approximately $100 \mu \mathrm{m}$ wide, were observed in the polymeric wall between adjacent pores, likely due to the SPS sintering step that notably allowed the presence of numerous tight contact bridges between $\mathrm{NaCl}$ grains. The biporous material (BP) thus exhibits the two different porosity levels in the same order of magnitude as those obtained for their monoporous counterparts.

As could be seen in Fig. 2, the SEM observation at higher magnification of the environment located between the two porosity networks allowed for distinguishing a thin $(\sim 500 \mathrm{~nm})$ and dense polymer film, previously shaped around the surface of 

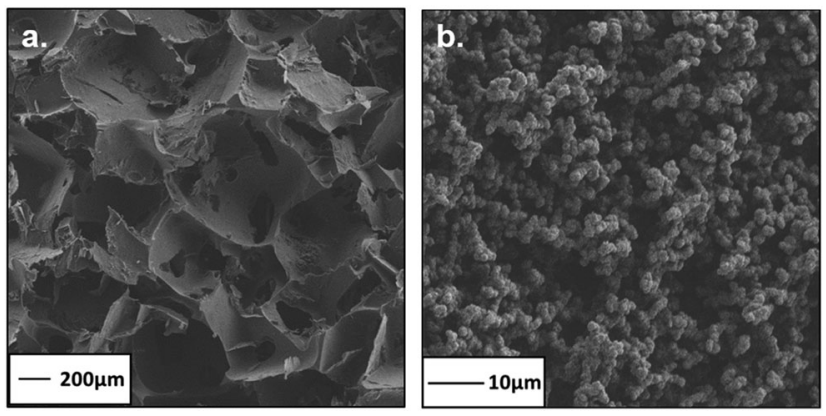

Fig. 1 SEM micrographs of $\mathrm{P}\left(\mathrm{HEMA}_{70}-\mathrm{CO}-\mathrm{EGDMA}_{30}\right)$ monoporous networks: (a) monoporous networks of large pores as imprint of the sintered 3-D $\mathrm{NaCl}$ template and (b) monoporous networks of small pores obtained after extraction of i-PrOH.

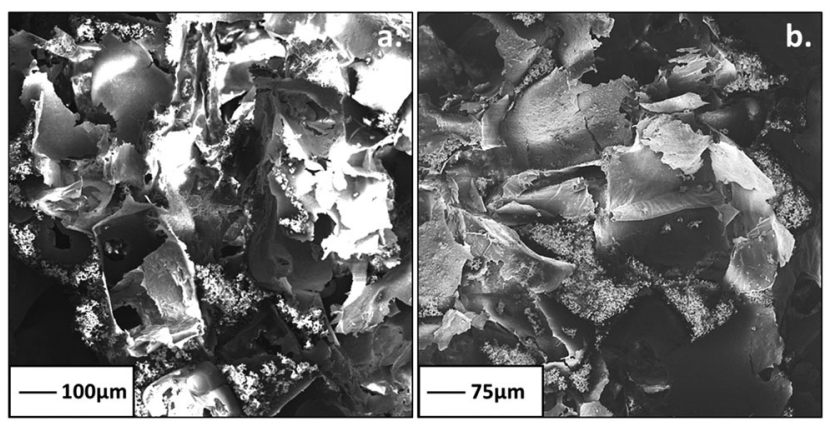

Fig. 2 (a) SEM micrographs of biporous $\mathrm{P}\left(\mathrm{HEMA}_{70}-\mathrm{CO}-\mathrm{EGDMA}_{30}\right)$ networks and (b) higher magnification of the environment between the different porosity levels.

the $\mathrm{NaCl}$ grains during the polymerization. The formation of this thin polymer film between the two porosity levels is likely due to interface concerns between the comonomer/solvent solution and the $\mathrm{NaCl}$ grains. Indeed, the homogeneous solution of $\mathrm{i}-\mathrm{PrOH}$ and comonomers probably suffered from a phase separation at the surface of the $\mathrm{NaCl}$ grain. Note the initiation sites of polymer nucleation, growing at the surface, to develop the lower porosity level. It was not obvious that this polymer layer isolated completely the two porosity levels. In fact, numerous gaps were expected to result from tension induced by the drying process, making transfers possible between the two porous phases. As shown in the following sections, this assumption was confirmed by imbibition results.

Density measurements were performed for the sintered 3-D templates and the polymeric samples, i.e. HEMA-based mono- and biporous materials and bulk HEMA-based material (prepared with 70 wt\% HEMA). The obtained values along with the theoretical porosity ratios are gathered in Table 1 . While pure $\mathrm{NaCl}$ displayed a density of $2.14 \mathrm{~g} \mathrm{~cm}^{-3}$, that of its corresponding sintered 3-D template was close to $1.84 \pm 0.06 \mathrm{~g} \mathrm{~cm}^{-3}$, that is to say it was composed of $85 \mathrm{vol} \%$ of salt and $15 \mathrm{vol} \%$ of pores. This empty space was then filled with co-monomers for preparing LP samples so as to theoretically reach $85 \%$ porosity. The density of the HEMA-based LP material was further measured and found to be equal to $0.17 \mathrm{~g} \mathrm{~cm}^{-3}$, i.e. $15 \%$ of the density of the dense, pure HEMA-based homopolymer.
Table 1 Theoretical porosity as determined via material's density

\begin{tabular}{llc}
\hline Compound & Density $\left(\mathrm{g} \mathrm{cm}^{-3}\right)$ & Porosity (vol\%) \\
\hline NaCl & 2.14 & 0 \\
NaCl 3D template & 1.84 & 15 \\
Bulk PHEMA (70 wt\% HEMA) & 1.17 & 0 \\
Large pores sample (LP) & 0.17 & 85 \\
Small pores sample (SP) & 0.28 & 76 \\
Biporous samples (BP) & 0.033 & 97
\end{tabular}

For the corresponding biporous material, the empty space left after sintering of the $\mathrm{NaCl} 3-\mathrm{D}$ template was filled with a solution of co-monomers and porogenic solvent (i-PrOH). This polymerization mixture composed of $80 \mathrm{vol} \% \mathrm{i}-\mathrm{PrOH}$ allows for producing the lower porosity level in the HEMA-based biporous material (around $5 \mu \mathrm{m}$ ). The density of the latter was found to be equal to $0.28 \mathrm{~g} \mathrm{~cm}^{-3}$, that is to say it presented a porosity ratio of $76 \mathrm{vol} \%$. Therefore, the theoretical porosity of the biporous material was equal to $0.85+0.15 \times 0.76=0.965$. This value was very close to the experimental value (see Table 1 ): $1-0.033 / 1.17=0.97$.

The presence of $\mathrm{NaCl}$ macroparticles did not modify the UV polymerisation in the presence of i-PrOH porogenic solvent. As shown in Fig. 3, unique porosity levels were observed for monoporous crosslinked polymers at $\sim 5 \mu \mathrm{m}$ and $100 \mu \mathrm{m}$ for polymeric materials obtained using the porogenic solvent (i-PrOH) and sieved sintered $\mathrm{NaCl}$ macroparticles, respectively. As expected from previous results, ${ }^{19}$ the pore distribution of the biporous polymers obtained by MIP displayed porosity levels identical to those from distinct monoporous polymeric frameworks. Indeed, two porosity levels were observed for biporous materials with the same size range using the double porogen templating approach. The $100 \mu \mathrm{m}$-sized higher porosity level obtained by MIP represents the diameter of the interconnections between adjacent pores, due to the threshold size theory. ${ }^{19,20}$

\subsection{Hydrophobicity of $\mathrm{P}(\mathrm{HEMA}-\mathrm{co}$-EgMA) polymeric materials}

Contact angle measurements were performed on polymer films incorporating different amounts of the EgMA monomer.

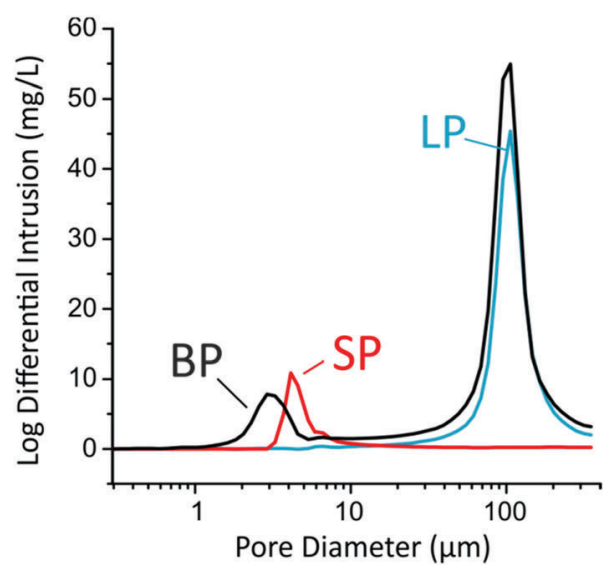

Fig. 3 Mercury intrusion porosimetry profiles showing the pore diameters of poly(2-hydroxyethyl methacrylate-co-ethyleneglycol dimethacrylate) monoporous samples, i.e. SP (small pores) and LP (large pores), and biporous (BP) samples. 

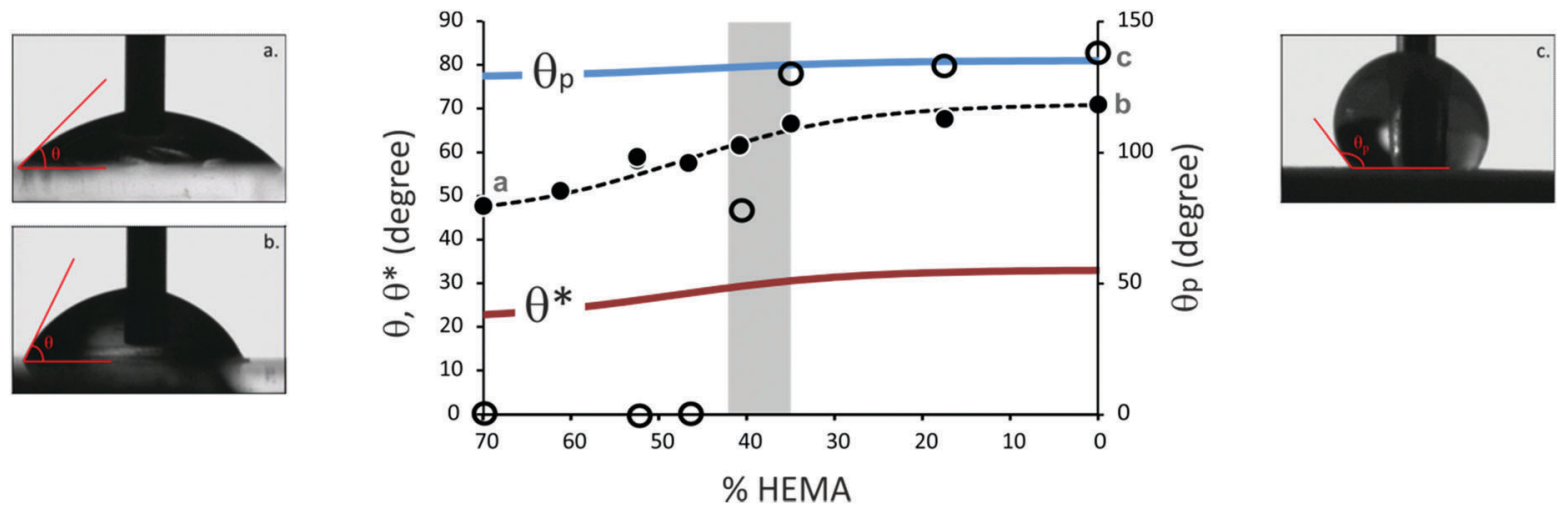

Fig. 4 Effect of HEMA content in the P(EGDMA EO-CO-HEMA $\left._{x}-\mathrm{CO}-\mathrm{EgMA}_{y}\right)$ copolymer on contact angle $\theta$ measured for water drops deposited on solid polymer films (full circles, e.g. (a) $\theta=48^{\circ}$, (b) $\theta=70^{\circ}$ ) and contact angle $\theta_{\mathrm{p}}$ measured for water drops deposited on the horizontal porous (SP) surface of samples (empty circles, e.g. (c) $\theta_{\mathrm{p}}=130^{\circ}$ ). Note that the value $\theta_{\mathrm{p}}=0$ means that the water drop invades the bulk sample porosity. The shaded area highlights the range of \%HEMA values where the transition $\theta_{p}=0 \rightarrow \theta_{p}=130^{\circ}$ is observed. Lines $\theta^{\star}$ and $\theta_{p}$ correspond to the theoretical values given by the Cassie-Baxter model for, respectively, polymer/water (eqn (8)) and polymer/air composite surfaces.

The partial or total substitution of the HEMA hydrophilic monomer by the hydrophobic EgMA monomer was shown to increase significantly the contact angle $\theta$ of the polymeric matrix (see Fig. 4). More precisely, $\theta$ is increased from $48^{\circ}$ for $\%$ HEMA $=70$ to $70^{\circ}$ for the fully substituted polymer. Contact angles $\theta_{\mathrm{p}}$ were also measured for water drops deposited on the surface of porous (SP) samples with several \%HEMA values (see Fig. 4). The major effect of HEMA substitution was found to induce a transition from a drop imbibition regime for $\%$ HEMA $\gtrsim 40$, for which a contact angle $\theta_{\mathrm{p}}=0^{\circ}$ has been set in Fig. 4, to a no-imbibition regime for \%HEMA $\lesssim 35$, characterized by a contact angle $\theta_{\mathrm{p}} \approx 130^{\circ}$.

\subsection{Permeability}

Permeability experiments performed on LP, SP and BP HEMAbased scaffolds have provided two ranges of values: $k=4 \pm 2 \times$ $10^{-11} \mathrm{~m}^{2}$ for LP and BP samples and $k=3 \pm 2 \times 10^{-13} \mathrm{~m}^{2}$ for the SP samples. We didn't measure any effect of \%HEMA on the permeability value, so in the following we will consider those values as constants. The permeability of SP samples was measured to be two orders of magnitude lower than those of LP and BP samples, thus demonstrating the crucial role of large pores in fluid flow across the polymeric porous materials. Although various semi-empirical approaches attempted to predict the permeability of porous systems, there exists only one clear reference model, corresponding to straight cylindrical channels of radius $R$, for which the permeability is $k_{0}=\phi R^{2} / 8$ and the permeability of a dense granular packing with beads of the same radius is typically about five times smaller. ${ }^{21,22}$ This provides a range $1.2 \times 10^{-13}-6 \times 10^{-13} \mathrm{~m}^{2}$, for the small pores and $1 \times 10^{-11}-10 \times 10^{-11} \mathrm{~m}^{2}$ for the large pores (taking respectively $R=5 \mu \mathrm{m}$ and $R=100 \mu \mathrm{m}$ ), which encompass our range of results for each material type.

\subsection{Imbibition properties}

Imbibition properties for the three types of porous material, namely the small pore (SP) and large pore (LP) monoporous and the biporous (BP) materials, synthesized with different chemical compositions, were measured. The corresponding imbibition curves are presented in Fig. 5. It is shown that both the pore size and the monoporous/biporous nature of the samples have significant effects on the imbibition rate: (1) imbibition of the LP sample is slower than the imbibition of the SP sample, and (2) imbibition of the biporous material is faster than that of both monoporous counterparts. In addition, all the samples show a decrease of imbibition rate as HEMA is substituted by EgMA.

\section{Discussion}

\subsection{Imbibition characteristics of the different porous media}

Let us start by briefly recalling the classical theory for capillary imbibition of liquids through porous media. By introducing the mean curvature of the water interface in the pores, $1 / \bar{r}$, the driving capillary pressure is given by the Laplace law: $2 \sigma / \bar{r}$. The progression of the interface at height $h$ is restrained by gravity, so the effective driving pressure is $\Delta P=2 \sigma / \bar{r}-\rho g h(t)$. The resulting interface velocity can be estimated via the Darcy law: $V_{\mathrm{D}}=k / \mu \times \Delta P / h$, where $V_{\mathrm{D}}=\phi \mathrm{d} h / \mathrm{d} t$ is the Darcy velocity. The equation for the evolution of the interface height is therefore:

$$
v_{\mathrm{I}} \equiv \frac{\mathrm{d} h}{\mathrm{~d} t}=\frac{2 \sigma k}{\phi \mu \bar{r}} \frac{1}{\phi}-\frac{\rho g k}{\phi \mu}=\frac{a}{h}-b
$$

where parameters $a=\frac{2 \sigma k}{\phi \mu \bar{r}}$ and $b=\frac{\rho g k}{\phi \mu}$ have been introduced. Note that the ratio $a / b$ corresponds to the Jurin height: $h_{\infty}=2 \sigma / \rho g \bar{r}$. The solution of eqn (3) is given by: ${ }^{7}$

$$
t=-\frac{h}{b}-\frac{a}{b^{2}} \ln \left(1-\frac{b h}{a}\right)
$$

Note also that eqn (3) simplifies for $h \ll a / b$ and the Washburn solution can be conveniently used:

$$
v_{\mathrm{I}} \equiv \frac{\mathrm{d} h}{\mathrm{~d} t} \cong \frac{a}{h}=\frac{2 \sigma k}{\phi \mu \bar{r}} \frac{1}{h}
$$



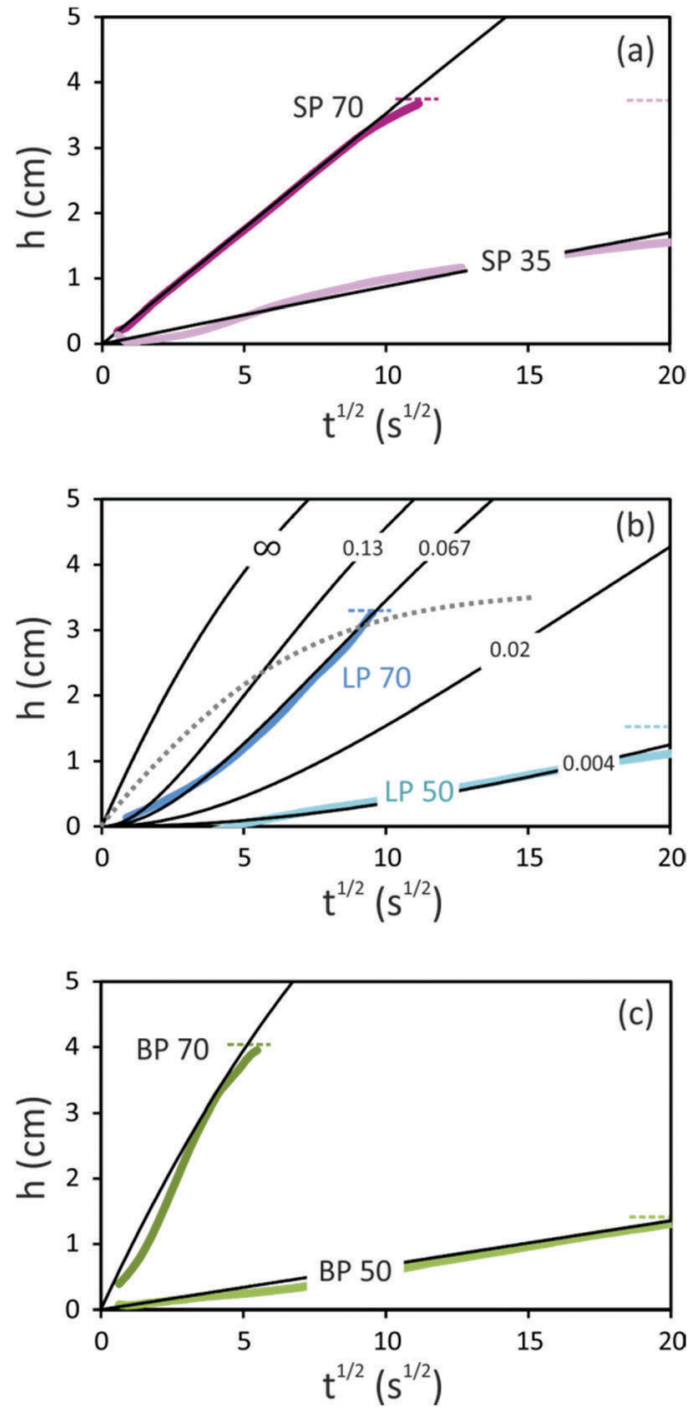

Fig. 5 Imbibition curves, i.e. water imbibition height as a function of square-root of time. Horizontal dotted lines indicate the height of the studied samples. (a) Monoporous small-pore materials containing $70 \%$ (SP70) and 35\% (SP35) HEMA. Black lines correspond to eqn (4) with parameters $\phi=0.76, k=3 \times 10^{-13} \mathrm{~m}^{2}, \bar{r}=9 \mu \mathrm{m}$ (SP70) and $\phi=0.76$, $k=3 \times 10^{-13} \mathrm{~m}^{2}, \bar{r}=140 \mu \mathrm{m}$ (SP35). (b) Monoporous large-pore materials containing 70\% (LP70) and 50\% (LP50) HEMA. The dotted line corresponds to eqn (4) with parameters $\phi=0.85, k=4 \times 10^{-11} \mathrm{~m}^{2}, \bar{r}=400 \mu \mathrm{m}$. For continuous lines, it is assumed that the water interface spends an average time $t_{\mathrm{c}}=t_{0}\left(1+p h / t_{0}\right)$ at each connection between two neighboring pores during the vertical motion. The average time for the interface to travel a length $D_{\mathrm{p}}$ is therefore equal to $D_{\mathrm{p}} / v_{1}+t_{\mathrm{c}}$. Black lines correspond to eqn (7) with $v_{1}$ given by eqn (4) with parameters $\phi=0.85, k=4 \times 10^{-11} \mathrm{~m}^{2}$, $\bar{r}=150 \mu \mathrm{m}$. The different curves correspond to the values reported for $D_{\mathrm{p}} / t_{0}$ (in $\mathrm{cm} \mathrm{s}^{-1}$ ) and $p / t_{0}=1 / 3 \mathrm{~cm}^{-1}$. (c) Biporous materials containing 70\% (BP70) and 50\% (BP50) HEMA. Black line BP70 corresponds to eqn (4) with parameters $\phi=0.97, k=4 \times 10^{-11} \mathrm{~m}^{2}, \bar{r}=150 \mu \mathrm{m}$. Black line BP50 is obtained by assuming that imbibition of the porous skeleton (SP50 matrix) is the kinetically limiting process: eqn (4) is used with parameters: $\phi=0.76$, $k=3 \times 10^{-13} \mathrm{~m}^{2}, \bar{r}=160 \mu \mathrm{m}$.

$$
h \cong(2 a t)^{1 / 2}=\left(\frac{4 \sigma k}{\phi \mu \bar{r}} t\right)^{1 / 2}
$$

Now we analyze the imbibition curves obtained with the composition \%HEMA $=70$. We start with the monoporous sample SP70. Eqn (4) is fitted to the experimental curve (see Fig. 5a) in order to determine $\bar{r}$ while parameter $k$ is set to the measured value, i.e. $k=3 \times 10^{-13} \mathrm{~m}^{2}$. We obtain $\bar{r} \approx 9 \mu \mathrm{m}$, which appears to be a consistent value with regard to the estimated pore size, and it will be discussed in more detail in the following.

For the monoporous sample LP70, our results show so slow an imbibition rate that they cannot be described by eqn (4). Indeed, we have increased the value of $\bar{r}$ in eqn (4) in order to reduce the front velocity, but for values $\bar{r}>400 \mu \mathrm{m}$ the Jurin height becomes smaller than the observed front heights (see Fig. 5b). In order to identify the mechanism leading to such a discrepancy with theory, we recorded movies from the magnified surface of the sample (see Fig. 6). This revealed a halting pore-filling process: from pore to pore, the capillary ascending motion pauses regularly, and the durations of those pauses appear to be distributed within the range 0.1-3 s.

It is recognized that interface motions can be slowed down or even stopped by passing through pore junctions or through capillaries with significant expansion of the cross-section. ${ }^{23-25}$ We therefore attribute the observed pauses to the connection areas between pores, where the interface is expected to flatten, i.e. $\bar{r} \approx \infty$, for satisfying contact angle conditions at the sharp-edge constriction geometry. ${ }^{24}$ This means that the driving capillary pressure drops at constrictions and then spreading of the thin precursor film emanating from the rising liquid promotes the sudden pull-up of the interface in the new pore, where equilibrium contact angle conditions can be recovered. This process takes the time identified as the pauses during imbibition (see Fig. 6). We propose to account for the effect of the pauses by introducing the average duration $t_{\mathrm{c}}$ spent by the interface between two neighboring pores during the vertical motion (see Fig. 7b). The dynamics of the vertical motion between two successive stops can still be considered as mainly governed by the balance between capillary effects and Darcy's law (leading to eqn (4) and (5)). Therefore, the average time for the interface to travel a length $D_{\mathrm{p}}$ is equal to $D_{\mathrm{p}} / v_{\mathrm{I}}+t_{\mathrm{c}}$, and the modified imbibition velocity is given by:

$$
v_{\mathrm{I}, t_{\mathrm{c}}}=\frac{D_{\mathrm{p}}}{\frac{D_{\mathrm{p}}}{v_{\mathrm{I}}}+t_{\mathrm{c}}}=\frac{v_{\mathrm{I}}}{1+\frac{v_{\mathrm{I}}}{D_{\mathrm{p}} / t_{\mathrm{c}}}}
$$

In the following we chose to consider $t_{\mathrm{c}}$ as a fitting parameter such as $t_{\mathrm{c}}=t_{0}\left(1+p h / t_{0}\right)$, where $t_{0}$ is the minimal time and coefficient $p$ accounts for the slowdown effect of gravity (one can expect that time $t_{\mathrm{c}}$ increases as a function of height). Parameters $t_{0}$ and $p$ can be determined by fitting eqn (7) to our results. In practice, we choose $h_{i}$ values within the range of interest and we calculate the corresponding times $t_{i}=t_{i-1}+\left(h_{i}-h_{i-1}\right) /\left(v_{\mathrm{I}, t_{\mathrm{c}}}\right)_{i}$, where $\left(h_{i}-h_{i-1}\right)$ correspond to small steps. Note that the parameter $\bar{r}$ has only a weak effect on these curves, i.e. in this approach the major effect is due to $t_{\mathrm{c}}$, so we set $\bar{r}=150 \mu \mathrm{m}$, which is consistent with the pore size in this sample and, as shown in the following, it is also the value obtained for the 

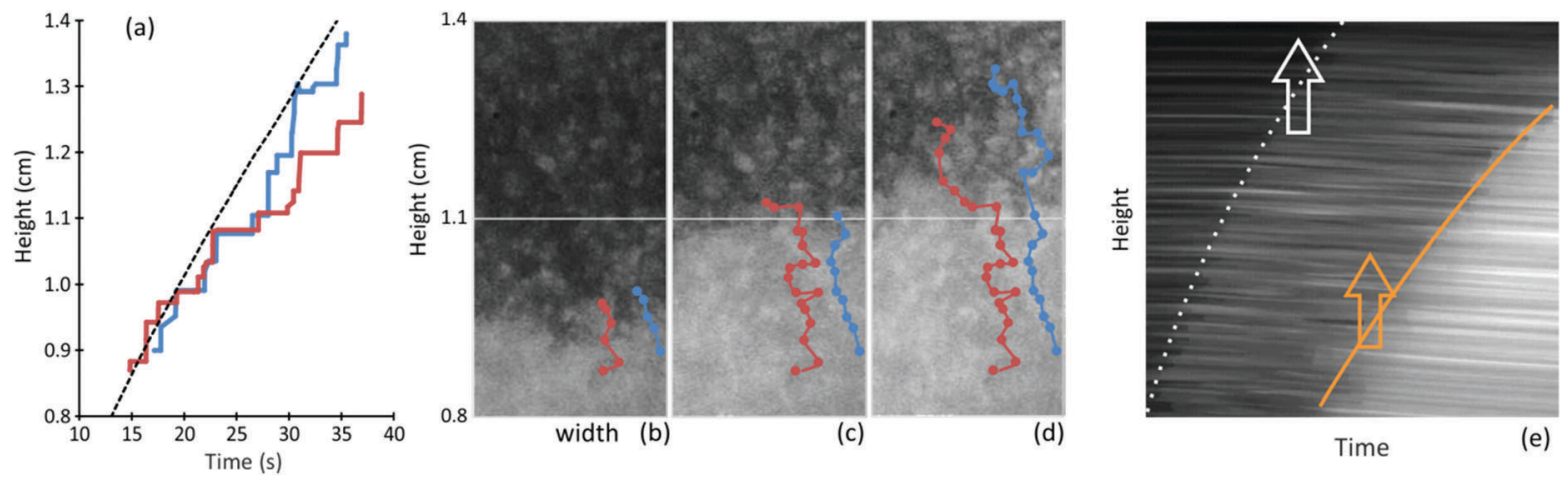

Fig. 6 Results for the kinetics of water imbibition as observed on a microscopic scale at sample's surface. (a) Positions of pores being included within two distinct imbibition paths through the LP samples as a function of time. The imbibition paths are presented in figures (b-d) with the same vertical length scale. Imbibition proceeds from pore to pore by "jumps" and "pauses" whose time averaging provides a macroscopic imbibition rate that is significantly smaller than the characteristic rate for the large pores. Duration of the "pauses" is measured to spread within the range $0.1-3 \mathrm{~s}$ and the average value is close to $0.5 \mathrm{~s}$. (e) Space-time picture (height: $1.1 \mathrm{~cm}$, time: $17 \mathrm{~s}$ ) for imbibition of biporous samples, i.e. the same vertical line within the sample's image is followed as a function of time. Note that under our conditions of light transmission through the sample, the light intensity level accounts for the degree of filling of the material by water. It reveals that two rising fronts develop in biporous samples, where the first one (dotted line) is attributed to imbibition of the small pore skeleton, and the second one (continuous) to the imbibition of the large pores.

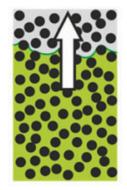

(a)

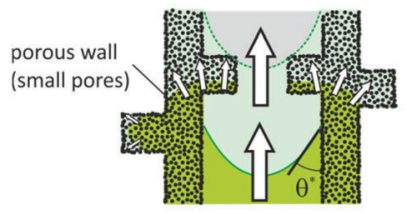

(c)

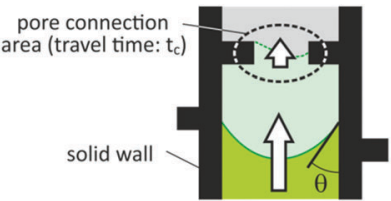

(b)

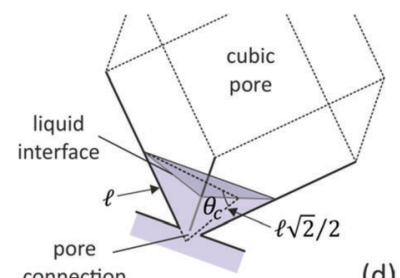

(d)
Fig. 7 Illustrations of the mechanisms involved in the imbibition of (a) small pores (SP), (b) large pores (LP) and (c) biporous (BP) samples. Note for the biporous material the presence of two rising liquid fronts: one into the small pore skeleton, and one in the large pore network. (d) Geometrical configuration of a flat liquid interface in a cubic pore. In such a pore geometry the interface motion is not allowed for contact angles $\theta$ larger than $\theta_{\mathrm{c}}$, where $\theta_{\mathrm{c}}$ is given by $\tan \theta_{\mathrm{c}}=\ell /(\ell \sqrt{2} / 2) \approx 75^{\circ}$.

biporous material. In Fig. $5 \mathrm{~b}$, eqn (7) with values $D_{\mathrm{p}} / t_{0}=$ $0.067 \mathrm{~cm} \mathrm{~s}^{-1}$ (i.e. $t_{0} \approx 0.6 \mathrm{~s}$ ) and $p / t_{0}=1 / 3 \mathrm{~cm}^{-1}$ shows good agreement with experimental results. Note that the small value $p / t_{0}$ indicates that the initial value $D_{\mathrm{p}} / t_{0}$ is the main parameter under our experimental conditions. Several curves are plotted with $D_{\mathrm{p}} / t_{0}$ values ranging from $\infty$ to $0.004 \mathrm{~cm} \mathrm{~s}^{-1}\left(t_{0}\right.$ ranging from 0 to $10 \mathrm{~s}$ respectively) keeping $p / t_{0}$ equal to $1 / 3 \mathrm{~cm}^{-1}$.

The biporous sample BP70 is now analyzed with eqn (4), showing good agreement by using the value $\bar{r}=150 \mu \mathrm{m}$. This indicates that imbibition proceeds through a standard continuous pore-filling process in the biporous sample. Eqn (7) can be used as well to describe our data under the condition of setting the value $D_{\mathrm{p}} / t_{0}>1$, which corresponds to $t_{0}<0.02 \mathrm{~s}$, i.e. the theoretical time spent by the interface at constrictions is not significant. This drastic evolution with respect to sample LP70 shows that the small pores, that are present within the polymer skeleton of the biporous material, contribute to neutralize the sharp-edge constriction effect described above.

The basic idea for understanding such a behavior is to consider that water can be transferred from the large pores to the porous skeleton, allowing for a synergistic mechanism to be set up. In the following we assume that walls of large pores are composite surfaces (polymer and air). Let us estimate the invasion velocity of the small pores by water coming from the large pores. The key point is to consider that for such a local imbibition process, liquid is available in the neighboring large pores, so the relevant value for $h$ in eqn (5) is $h \approx D_{\text {p }}$, leading to $v_{\mathrm{I}, \ell} \cong v_{\mathrm{I}}\left(h=D_{\mathrm{p}}\right) \approx 2 \sigma k / \phi D_{\mathrm{p}} \mu \bar{r} \approx 2 \mathrm{~cm} \mathrm{~s}^{-1}$. This value is by far larger than the measured imbibition rate for the BP70 sample, suggesting that the kinetically limiting process is the capillary rise in the network of large pores. As the porous skeleton is imbibed, the contact angle evolves for the water interface in the large pore network. This allows the pause time to be reduced to a low value.

Let us now examine more precisely the impact of the porous skeleton imbibition on its wetting properties. We stress that walls are composite surfaces, here a mixture of water and polymer, so the effective contact angle $\theta^{*}$ is expected to depend on both $\theta$ and the porosity of the composite surface, $\varphi$, as predicted by the Cassie-Baxter model ${ }^{26}$ for the polymer/water composite surface:

$$
\cos \left(\theta^{*}\right)=\cos \theta-\varphi(\cos \theta-1)
$$

Using $\theta=45^{\circ}$ and $\varphi=0.76$ (SP porosity) in eqn (8) provides the value $\theta^{*}=22^{\circ}$. Note that walls of the large pores in BP samples (see Fig. 2a) were found to be partially covered with a thin solid layer, which is expected to decrease this estimated value: $\theta^{*}=35^{\circ}$ is obtained with the assumption of $50 \%$ of wall surface covered with the solid layer (i.e. the value $\varphi=0.5 \times 0.76$ is used in eqn (8)). These two values indicate that the effective contact 
angle can be significantly lower than $\theta$, which is expected to promote the interface progression within the large pore network. Note also that invasion of the skeleton is expected to contribute to bypass the large pore connections (see Fig. 7c for an illustration). By using eqn (5), we estimate the time required for the interface to bypass the connection thickness, i.e. $0.1 D_{\mathrm{p}}$, through the small pore medium: $t_{\text {bypass }} \approx 0.1 D_{\mathrm{p}}{ }^{2} \phi \mu \bar{r} / 2 \sigma k \approx 0.002 \mathrm{~s}$. This estimated value falls within the range for which eqn (7) predicts that there is no appreciable slowdown due to the large pore connections, which is consistent with our observations: the pore wetting process involved in the excess time $t_{\mathrm{c}}$ for the LP sample is not required for the BP sample due to water flowing from the small pore skeleton.

\subsection{Impact of wetting characteristics of the porous skeleton}

Here we analyze the effect of the ratio \%HEMA. We emphasize that $\bar{r}$ accounts not only for the pore size but also for the conditions of contact for the interface at the walls in the porous medium, i.e. both the contact angle and the geometry of the solid walls. Therefore, for a given porous morphology, the evolution of $\bar{r}$ as a function of \%HEMA is expected to account for the effect of contact angle variation.

Using eqn (4) for sample SP35, we obtain $\bar{r}=140 \mu \mathrm{m}$ (see Fig. 5a). This clearly shows that the decrease observed for the imbibition rate is due to the drastic reduction of the driving capillary force. This is true also for the other monoporous sample, for which eqn (4) cannot be applied directly. Turning to eqn (7) provides a value for $D_{\mathrm{p}} / t_{0}$ that corresponds to $t_{0} \approx 10 \mathrm{~s}$ (see Fig. 5b). This reflects the weakening of driving capillary forces due to coupled effects of geometry, i.e. connections paths, and contact angle. The biporous material BP50 also is drastically influenced by contact angle. Using $\theta=60^{\circ}$ in eqn (8) provides the values $\theta^{*}=30^{\circ}\left(52^{\circ}\right.$ with the assumption of $50 \%$ of wall surface covered with the solid layer). These values are not so different than the values discussed above for the BP70 sample, so the contact angle at the walls of large pores cannot be responsible for the strong decrease of the imbibition rate. In fact, the main difference lies in the invasion velocity of the porous skeleton, $v_{\mathrm{I}, \ell}$, which is reduced by more than one order of magnitude (compare SP70 and SP35 in Fig. 5a). Therefore, it seems that the imbibition rate of the porous skeleton is the kinetically limiting process for the BP50 sample, i.e. the large pores fill at a velocity which is set by the imbibition rate of the skeleton, resulting in the strong decrease of imbibition rate with respect to BP70. As shown in Fig. 5c, the imbibition curve for BP50 is reasonably described, indeed, by eqn (4) using imbibition parameters for the SP medium, i.e. $k=3 \times 10^{-13} \mathrm{~m}^{2}$ and $\bar{r}=160 \mu \mathrm{m}$.

As described above, all the porous materials exhibit a strong decrease of the imbibition rate as \%HEMA decreases, or equivalently as $\theta$ increases. This behavior is summarized in Fig. 8 (inset) by plotting the average velocity $\Delta h / \Delta t$ (measured over the first $1 \mathrm{~cm}$ of front rise) as a function of $\theta$. One can see that the imbibition velocity of BP materials is larger than the sum of velocities measured for the monoporous samples. Besides, there exists a critical $\theta$ value above which the imbibition

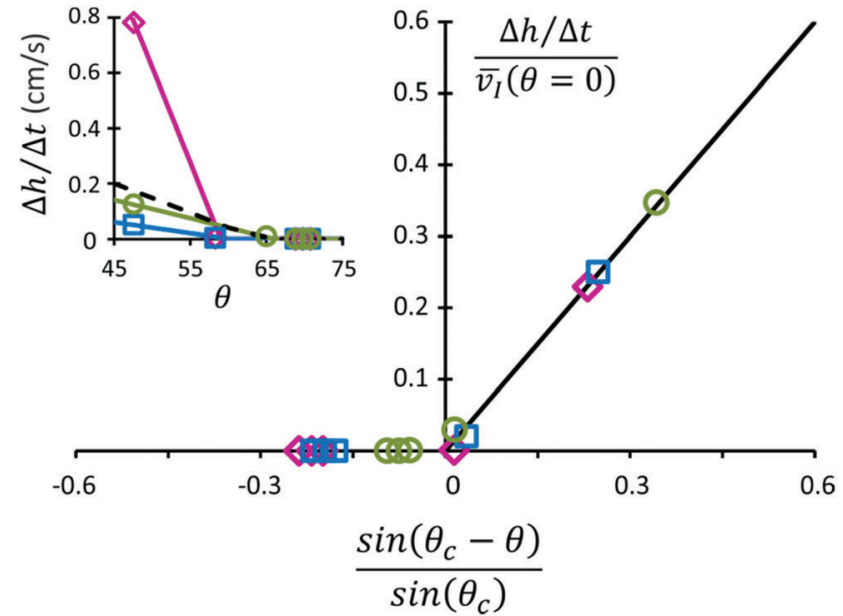

Fig. 8 Effect of contact angle on the imbibition rate for the studied materials: SP (circles), LP (squares) and BP (diamonds). Inset: Average imbibition velocity $\Delta h / \Delta t$ measured for $h=1 \mathrm{~cm}$ as a function of contact angle. Continuous lines correspond to data calculated by the relation: $\Delta h / \Delta t=\bar{v}_{\mid}(\theta=0) \times \sin \left(\theta_{c}-\theta\right) / \sin \left(\theta_{c}\right)$ where parameters $\bar{v}_{\mid}(\theta=0)$ and $\theta_{c}$ are introduced in the main text and are determined by plotting the main figure. The dashed line corresponds to the sum of velocities obtained with LP and SP samples. Main figure: Reduced velocity as a function of the difference between contact angle and the critical contact angle $\theta_{c}$ (below which water imbibes the material). For each type of sample (i.e. SP, LP and BP), parameters $\bar{v}_{l}(\theta=0)$ and $\theta_{c}$ have been fitted in order to approach as much as possible the theoretical straight line with a slope of unity.

rate is zero, i.e. water does no more spontaneously imbibe the porous samples. This has also been revealed by contact angle measurements for water drops on porous surfaces (see Fig. 4): drops imbibe the porous medium for smaller $\theta$ values but they remain on the sample's surface for larger $\theta$ values. For the latter case, the sample's surface behaves as a solid composite surface (polymer/air) and the effective contact angle is given by the Cassie-Baxter model: $\cos \left(\theta_{\mathrm{p}}\right)=\cos \theta-\varphi(\cos \theta+1)$. Using $\theta=$ $60^{\circ}$ and $\varphi=0.76$ provides the value $\theta_{\mathrm{p}}=130^{\circ}$, which is in very good agreement with the measured value (see Fig. 4). Therefore, the no-imbibition regime is related to a critical value $\theta_{\mathrm{c}}$ that is expected to depend on the microscopic features of the porosity.

In the classical model of straight vertical capillaries of radius $R$, the capillary radius is given by $\bar{r}=R / \cos (\theta)=R / \sin (\pi / 2-\theta)$, where $\theta_{\mathrm{c}}=\pi / 2$ is the critical contact angle: for such a value of the contact angle, the interface is flat, i.e. $\bar{r}=\infty$, and water does not rise in the straight capillary if $\theta \geq \pi / 2$. For more complex pore geometries exhibiting edges and vertexes, the critical contact angle $\theta_{c}$ can be significantly different than $\pi / 2$. For a cubic pore, (1) the liquid cannot invade the pore spontaneously through an aperture located through a face for any finite value of the contact angle (this would involve a positive Laplace pressure for the interface); (2) the contact angle has to be smaller than $45^{\circ}$ for invading the pore through an aperture located in an edge; ${ }^{27}$ (3) the most favorable configuration corresponds to the smallest expansion ratio of the pore crosssection (i.e. the interface enters into the pore through a cornersee Fig. 7d) and the associated critical contact angle value can be calculated by considering the flat interface configuration 
presented in Fig. 7: $\theta_{\mathrm{c}} \approx 75^{\circ}$. Therefore, with regard to the measured contact angles on the polymer surfaces, i.e. $\theta \in\left[48-70^{\circ}\right]$, the cubic pores are expected to be invaded mostly from the corners. On the other hand, biporous sample BP70 revealed a two-step imbibition process, where imbibition of the small pore skeleton is expected to promote the imbibition of the large pores by decreasing the water contact angle at the large pore walls to a value potentially smaller than $45^{\circ}$. As a result, many imbibition paths (i.e. corner and edge apertures) are expected to be available in the BP70 sample, which is expected to contribute to increase the imbibition rate with respect to the LP70 sample.

As the imbibition velocity is inversely proportional to $\bar{r}$, a simple way for identifying $\theta_{\mathrm{c}}$ and $R$ for the studied samples is to plot the evolution of the imbibition velocity as a function of $\sin \left(\theta_{c}-\theta\right)$. By introducing the imbibition velocity obtained for $\theta=0$, i.e. the maximum imbibition velocity $\bar{v}_{\mathrm{I}}(\theta=0)$, and the critical contact angle $\theta_{\mathrm{c}}$ as fitting parameters, a plot $(\Delta h / \Delta t) / \bar{v}_{\mathrm{I}}(\theta=0)$ as a function of $\sin \left(\theta_{\mathrm{c}}-\theta\right) / \sin \left(\theta_{\mathrm{c}}\right)$ should exhibit a linear dependence with a slope of unity for $\theta<\theta_{\mathrm{c}}$, i.e. for $\sin \left(\theta_{\mathrm{c}}-\theta\right)>0$. Fig. 8 shows such a plot where $\bar{v}_{\mathrm{I}}(\theta=0)$ and $\theta_{c}$ have been fitted in order to approach as much as possible a linear evolution for each type of porous material, respectively: $0.36 \mathrm{~cm} \mathrm{~s}^{-1}$ and $65^{\circ}$ for $\mathrm{SP}, 0.20 \mathrm{~cm} \mathrm{~s}^{-1}$ and $60^{\circ}$ for $\mathrm{LP}$, and $3.4 \mathrm{~cm} \mathrm{~s}^{-1}$ and $59^{\circ}$ for BP. Note that the average velocity obtained for the small pore sample is consistent with the theoretical value discussed previously, i.e. $\bar{v}_{\mathrm{I}}(\theta=0, h)=4 \sigma k / \phi \mu \bar{r} h \approx$ 0.4 using values $k=3 \times 10^{-13} \mathrm{~m}^{2}$ and $\bar{r}=3 \mu \mathrm{m}$, i.e. $R \approx 3.3 \mu \mathrm{m}$. This value is in good agreement with the pore size presented in Fig. 3. These results show that (i) imbibition in SP samples is promoted by a slightly higher value for the critical contact angle $\theta_{\mathrm{c}}$, i.e. for a given $\theta$ value the difference $\left(\theta_{\mathrm{c}}-\theta\right)$ is larger - so is the driving capillary force, and (ii) for $\theta=0$, the imbibition rate of BP samples is intrinsically larger than those for their monoporous counterparts (see for example the inset in Fig. 8).

\section{Conclusions}

We have studied water absorption in porous organic materials exhibiting several degrees of hydrophobicity and two pore size levels, either as monoporous materials (large or small pores) or as biporous materials (mixed large and small pores). Absorption for the small pore material is well described by the classical imbibition theory, in which the driving capillary pressure is set by both the pore size, i.e. several micrometers, and the solid/liquid contact angle. Large pore materials showed strongly reduced imbibition rates with respect to the theoretical value deduced from their pore size. This behavior has been shown to result from the excess time spent by the liquid interface within the connection areas that separate neighboring pores. Remarkably, this connection-limited imbibition regime is completely suppressed for the biporous material and the imbibition rate is even higher than the sum of rates obtained for its monoporous counterparts. This strong synergistic action of mixed small and large pores has been shown to be related to the liquid transfer allowed between the two porosities: critical crossing points within the interconnected large pore network can be bypassed through the small pore network. This result highlights the potential of hierarchical porous materials to be designed with dedicated imbibition properties.

\section{Conflicts of interest}

There are no conflicts to declare.

\section{Acknowledgements}

This work has benefited from a French government grant by ANR within the framework of the national program Investments for the Future ANR-11-LABX-022-01 (LabEx MMCD project).

\section{References}

1 C. Ma and B. Chen, Properties of foamed concrete containing water repellents, Constr. Build. Mater., 2016, 123, 106-114, DOI: 10.1016/j.conbuildmat.2016.06.148.

2 Water Properties in Food, Health, Pharmaceutical and Biological Systems: ISOPOW 10., ed. D. S. Reid, T. Sajjaanantakul, P. J. Lillford and S. Charoenrein, Wiley-Blackwell, 2010.

3 J. Kim, M. W. Moon, K. R. Lee, L. Mahadevan and H. Y. Kim, Hydrodynamics of writing with ink, Phys. Rev. Lett., 2011, 107, 2-5, DOI: 10.1103/PhysRevLett.107.264501.

4 A. Naillon, H. Massadi, R. Courson, J. Bekhit, L. Seveno, P. F. Calmon, M. Prat and P. Joseph, Quasi-static drainage in a network of nanoslits of non-uniform depth designed by grayscale laser lithography, Microfluid. Nanofluid., 2017, 21, 1-14, DOI: 10.1007/s10404-017-1970-z.

5 J. M. Bell and F. K. Cameron, The flow of liquids through capillary spaces, J. Phys. Chem., 1906, 10, 658-674.

6 R. Lucas, Ueber das Zeitgesetz des kapillaren Aufstiegs von Flüssigkeiten, Kolloid-Z., 1918, 23, 15-22.

7 E. W. Washburn, The dynamics of capillary flow, Phys. Rev., 1921, 17, 273-283.

8 W. E. Brittin, Liquid Rise in a Capillary Tube, J. Appl. Phys., 1946, 17, 37 .

9 G. Mason and N. R. Morrow, Effect of Contact Angle on Capillary Displacement Curvatures in Pore Throats Formed by Spheres, J. Colloid Interface Sci., 1994, 130-141, DOI: 10.1006/jcis.1994.1402.

10 W. W. Liou, Y. Peng and P. E. Parker, Analytical modeling of capillary flow in tubes of nonuniform cross section, J. Colloid Interface Sci., 2009, 333, 389-399, DOI: 10.1016/ j.jcis.2009.01.038.

11 D. Erickson, D. Li and C. B. Park, Numerical simulations of capillary-driven flows in nonuniform cross-sectional capillaries, J. Colloid Interface Sci., 2002, 250, 422-430, DOI: $10.1006 /$ jcis.2002.8361. 
12 M. Alava, M. Dubé and M. Rost, Imbibition in Disordered Media, 2004, https://arxiv.org/pdf/cond-mat/0402201.pdf (accessed June 29, 2018).

13 S. Gruener, Z. Sadjadi, H. E. Hermes, A. V. Kityk, K. Knorr, S. U. Egelhaaf, H. Rieger and P. Huber, Anomalous front broadening during spontaneous imbibition in a matrix with elongated pores, Proc. Natl. Acad. Sci. U. S. A., 2012, 109, 10245-10250, DOI: 10.1073/pnas.1119352109.

14 E. K. K. Nambiar and K. Ramamurthy, Sorption characteristics of foam concrete, Cem. Concr. Res., 2007, 37, 1341-1347, DOI: 10.1016/j.cemconres.2007.05.010.

15 M. Zhou, S. Caré, D. Courtier-Murias, P. Faure, S. Rodts and P. Coussot, Magnetic resonance imaging evidences of the impact of water sorption on hardwood capillary imbibition dynamics, Wood Sci. Technol., 2018, 52, 929-955, DOI: 10.1007/s00226-018-1017-y.

16 L. Rojo, B. Vazquez, J. Parra, A. López Bravo, S. Deb and J. San Roman, From Natural Products to Polymeric Derivatives of "Eugenol": A New Approach for Preparation of Dental Composites and Orthopedic Bone Cements, Biomacromolecules, 2006, 7, 2751-2761.

17 J. Deng, B. Yang, C. Chen and J. Liang, Renewable Eugenol-Based Polymeric Oil-Absorbent Microspheres: Preparation and Oil Absorption Ability, ACS Sustainable Chem. Eng., 2015, 3, 599-605.

18 J. Yang, M. Khan, L. Zhang, X. Ren, J. Guo, Y. Feng, S. Wei and W. Zhang, Antimicrobial surfaces grafted random copolymers with REDV peptide beneficial for endothelialization, J. Mater. Chem. B, 2015, 3, 7682-7697.
19 H. B. Ly, B. Le Droumaguet, V. Monchiet and D. Grande, Facile fabrication of doubly porous polymeric materials with controlled nano- and macro-porosity, Polymer, 2015, 78, 13-21.

20 H. B. Ly, B. Le Droumaguet, V. Monchiet and D. Grande, Designing and modeling doubly porous polymeric materials, Eur. Phys. J.: Spec. Top., 2015, 224, 1689-1706.

21 J. Kozeny, Ueber kapillare Leitung des Wassers im Boden, Sitzungsber Akad. Wiss., Wien., 1927, 136, 271-306.

22 P. C. Carman, Fluid flow through granular beds, Trans. Inst. Chem. Eng., 1937, 15, 150-166, DOI: 10.1016/S0263-8762(97) 80003-2.

23 H. S. Wiklund and T. Uesaka, Microfluidics of imbibition in random porous media, Phys. Rev. E: Stat., Nonlinear, Soft Matter Phys., 2013, 87, 023006.

24 H. Mehrabian, P. Gao and J. J. Feng, Wicking flow through microchannels, Phys. Fluids, 2011, 23, 1-14, DOI: 10.1063/ 1.3671739 .

25 Z. Sadjadi, M. Jung, R. Seemann and H. Rieger, Meniscus arrest during capillary rise in asymmetric microfluidic pore junctions, Langmuir, 2015, 31, 2600-2608, DOI: 10.1021/ la504149r.

26 A. B. D. Cassie and S. Baxter, Wettability of porous surfaces, Trans. Faraday Soc., 1944, 546-551.

27 S. Herminghaus, M. Brinkmann and R. Seemann, Wetting and Dewetting of Complex Surface Geometries, Annu. Rev. Mater. Res., 2008, 38, 101-121, DOI: 10.1146/annurev. matsci.38.060407.130335. 